\title{
Uniform Euler approximation of solutions of fractional-order delayed cellular neural network on bounded intervals
}

\author{
Swati Tyagi ${ }^{1}$, Syed Abbas $^{1 *}$, Manuel Pinto ${ }^{2}$ and Daniel Sepúlveda ${ }^{3}$ \\ ${ }^{1}$ School of Basic Sciences, Indian Institute of Technology, Mandi, H.P., 175001, India. \\ ${ }^{2}$ Departamento de Matemáticas, Facultad de Ciencias, Universidad de Chile, Casilla 653, Santiago, Chile. \\ ${ }^{3}$ Escuela de Matemática y Estadística, Universidad Central de Chile, Toesca 1783, Santiago, Chile. \\ E-mail: sabbas.iitk@gmail.com*
}

\begin{abstract}
In this paper, we study convergence characteristics of a class of continuous time fractional-order cellular neural network containing delay. Using the method of Lyapunov and Mittag-Leffler functions, we derive sufficient condition for global Mittag-Leffler stability, which further implies global asymptotic stability of the system equilibrium. Based on the theory of fractional calculus and the generalized Gronwall inequality, we approximate the solution of the corresponding neural network model using discretization method by piecewise constant argument and obtain some easily verifiable conditions, which ensures that the discrete-time analogues preserve the convergence dynamics of the continuous-time networks. In the end, we give appropriate examples to validate the proposed results, illustrating advantages of the discrete-time analogue over continuous-time neural network for numerical simulation.
\end{abstract}

2010 Mathematics Subject Classification. 26A33. 92B20; 34K07; 34A45

Keywords. Fractional-order; Neural network; Mittag-Leffler stability; Approximate solution; Error analysis; Piecewise constant argument of generalized type.

\section{Introduction}

The Fractional-calculus as a generalization of integer order differentiation and integral to noninteger order was first introduced 300 years ago, though their applications are rather recent. It has wide range of applications in control theory [1], viscoelasticity [2], signal processing [3] and social sciences [4].

It has been recognized that the integral derivative of a function is related only to its nearby points, while fractional derivative is related to all of the function history. It can be interpreted in other way that the future state of the system depends on its present state as well as upon its history values starting from the initial point. Thus fractional-order model possesses memory, and due to this, it becomes precise to describe the state of the neurons. Fractional derivative has ability to model memory and hereditary properties, since the fractional-derivative is non-local. Thus the models containing fractional derivative are very crucial. Recently, it has been found that the fractional-order calculus could be used in the study of Hopfield neural networks. Various results on fractional-order neural networks have been obtained as a result of development of fractional calculus [5-8]. The discrete time fractional-order artificial neural networks were presented in $[9,10]$.

${ }^{*}$ Corresponding author 
Due to finite switching speeds of the amplifiers, time-delay is inevitable, which can also result in oscillations or instability of dynamic system. Thus, it becomes an interesting topic to study the stability of fractional-order neural networks containing delays. Also in some literatures, chaos and chaotic synchronization has been discussed [11-13].

In [14], Zhang et al. have investigated the Mittag-Leffler stability for a fractional-order neural network. In this paper, we study the Mittag-Leffler stability for a fractional-order neural network by introducing time delay term. Moreover, we derive sufficient approximating results for the solutions of fractional-order delayed neural network via solutions of corresponding fractional-order delayed neural network with piecewise constant arguments of generalized type. Having obtained sufficient conditions for the convergence dynamics of the continuous-time network, we propose an innovative method for obtaining discrete-time analogues from the continuous-time network. In particular, we propose a novel method to obtain a discrete-time fractional-order dynamical system whose dynamics is inherited from the continuous-time dynamic system. The discretization method proposed in this paper is different form the other discretization methods (such as predictor-corrector and Euler method), in the sense that our method is an approximation via piecewise constant arguments of generalized type, which has not been obtained in the related field of neural networks so far. Thus the results obtained are new and more general than the previous results derived in literature.

In this paper, we consider the following delayed fractional-order neural network model described by the following differential equation:

$$
\begin{aligned}
D_{t}^{\alpha} x_{i}(t) & =-a_{i} x_{i}(t)+\sum_{j=1}^{m} b_{i j} f\left(x_{j}(t)\right)+\sum_{j=1}^{m} c_{i j} g\left(x_{j}(t-\tau)\right)+I_{i} \quad t \in[0, T) \\
x_{i}(t) & =\varphi_{i}(t), \quad t \in[-\tau, 0], \quad T<\infty, \quad i=1,2, \cdots, m,
\end{aligned}
$$

where $m$ represents the number of units in the neural network; $D_{t}^{\alpha}$ denotes the Liouville-Caputa fractional derivative of order $\alpha, 0<\alpha \leq 1 ; x(t)=\left(x_{1}(t), x_{2}(t), \cdots, x_{m}(t)\right)^{T} \in \mathbb{R}^{m}$ corresponds to the state vector at time $t ; f($.$) and g($.$) denote the activation functions; I_{i}$ are constants denote the external bias on the $i^{t h}$ neuron; the positive constants $a_{i}>0$ denote the rates with which the $i^{t h}$ neuron will reset its potential to the resting state in isolation when disconnected from the networks and external inputs; the constants $b_{i j}$ and $c_{i j}$ denote the connections of $j^{\text {th }}$ neuron to $i^{\text {th }}$ neuron at time $t$ and $t-\tau ; \tau$ is the transmission delay respectively; $a_{i}=\operatorname{diag}\left(a_{1}, a_{2}, \cdots, a_{m}\right), b_{i j}, c_{i j}$ are the connection weight matrices; and $I_{i}=\left(I_{1}, I_{2}, \cdots, I_{m}\right)^{T}$ are external inputs. The initial conditions are $\varphi_{i}(t) \in C\left([-\tau, 0], \mathbb{R}^{m}\right)$, where $C\left([-\tau, 0], \mathbb{R}^{m}\right)$ denotes the Banach space of continuous $m$-real vector functions defined on $[-\tau, 0]$. $[p]$ denotes the integer part of $p$.

The structure of this paper is as follows. In Section 2, we give some preliminaries regarding fractional order differential systems. In Section 3, we establish the Mittag-Leffler stability of system (1.1) by constructing suitable Lyapunov function. In Section 4, using discretization scheme, we show that that the solution $z_{i}^{h}(t)$ of (4.2) approximates the solution $x_{i}(t)$ of (1.1) uniformly on any compact interval $[0, T), T<\infty$. Later, we prove that the error function converges to the actual solution for $h \rightarrow 0$ uniformly. Moreover, in Section 5, we present some numerical results to illustrate the effectiveness of the derived results followed with conclusion in Section 6. 


\section{Preliminaries on fractional - order differential systems}

For the convenience of reader, we recall some definitions and lemmas of fractional calculus in this section (for instance, see [15-17]).

Definition 2.1. [20] The Riemann-Liouville fractional integral with fractional-order $\alpha>0$ of function $f(t)$ is defined as

$$
{ }_{t_{0}} I_{t}^{\alpha} f(t)=\frac{1}{\Gamma \alpha} \int_{t_{0}}^{t}(t-s)^{\alpha-1} f(s) d s,
$$

where $\Gamma$ (.) is the Gamma function and is given by $\Gamma(z)=\int_{0}^{\infty} t^{z-1} e^{-t} d t$.

Definition 2.2. [19] The Liouville-Caputa derivative of fractional order $\alpha$ of a function $f(t)$ is defined as

$$
{ }_{C} D_{t_{0}, t}^{\alpha} f(t)=\frac{1}{\Gamma(n-\alpha)} \int_{t_{0}}^{t}(t-s)^{n-\alpha-1} f^{(n)}(s) d s
$$

where $n-1<\alpha<n \in \mathbb{Z}^{+}$.

Remark 2.3. For sake of simplicity, we denote

$$
{ }_{C} D_{t_{0}, t}^{\alpha} f(t)=D_{t}^{\alpha} f(t)
$$

Remark 2.4. When $\alpha \rightarrow 1$, the fractional order derivative $D_{t}^{\alpha} f(t)$ converges to the integer order derivative $f^{\prime}(t)$.

Definition 2.5. [19] The two-parameter Mittag-Leffler function is defined as:

$$
E_{\alpha, \beta}(z)=\sum_{k=0}^{\infty} \frac{z^{k}}{\Gamma(k \alpha+\beta)},
$$

where $\alpha>0, \beta>0$ and $z \in \mathbb{C}$, where $\mathbb{C}$ denotes the complex plane. When $\beta=1$, we have

$$
E_{\alpha, 1}(z)=E_{\alpha}(z)=\sum_{k=0}^{\infty} \frac{z^{k}}{\Gamma(k \alpha+1)}
$$

Further, for $\alpha=1, \beta=1$, we have $E_{1,1}(z)=e^{z}$.

Lemma 2.6. [19] The following properties exist for the Mittag-Leffler function.

1. There exists constants $N_{1}, N_{2} \geq 1$ such that for any $0<\alpha<1$,

$$
\begin{aligned}
\left\|E_{\alpha, 1}\left(A t^{\alpha}\right)\right\| & \leq N_{1}\left\|e^{A t}\right\|, \\
\left\|E_{\alpha, \alpha}\left(A t^{\alpha}\right)\right\| & \leq N_{2}\left\|e^{A t}\right\|,
\end{aligned}
$$

where $A$ denotes denotes matrix, $\|$.$\| denotes any vector or induced matrix norm.$ 
2. If $\alpha \geq 1$, then for $\beta=1,2, \alpha$

$$
\left\|E_{\alpha, \beta}\left(A t^{\alpha}\right)\right\| \leq\left\|e^{A t^{\alpha}}\right\|, \quad t \geq 0 .
$$

Consider an $n$-dimensional fractional-order system given by

$$
{ }_{t_{0}} D_{t}^{\alpha}=f(t, x(t)), \quad x\left(t_{0}\right)=x_{t_{0}} .
$$

Definition 2.7. (Generalized Mittag-Leffler stability [14]) The equilibrium point $x^{*}=\left(x_{1}^{*}, x_{2}^{*}, \cdots, x_{m}^{*}\right)^{T}$ of system (2.8) is said to be globally Mittag-Leffler stable, if

$$
\left\|x(t)-x^{*}\right\| \leq\left[m\left(x_{t_{0}}-x^{*}\right) E_{\alpha}\left(-\gamma\left(t-t_{0}\right)^{\alpha}\right)\right]^{b}
$$

where $\gamma>0, b>0, m(0)=0, m(x) \geq 0$ and $m(x)$ satisfies locally Lipschitz condition on $x \in \mathbb{R}^{m}$ with Lipschitz constant $m_{0}$.

Remark 2.8. The global Mittag-Leffler stability implies global asymptotic stability.

Theorem 2.9. (The Second Method of Lyapunov [14]) For $t_{0}=0$, the fractional-order system (2.8) is Mittag-Leffler stable at the equilibrium point $x^{*}=0$, if there exists a continuously differentiable function $V(t, x(t))$ satisfying

$$
\begin{array}{r}
\alpha_{1}\|x\|^{a} \leq V(t, x(t)) \leq \alpha_{2}\|x\|^{a b}, \\
D_{t}^{\beta} V(t, x(t)) \leq-\alpha_{3}\|x\|^{a b},
\end{array}
$$

where $V(t, x(t)):[0, \infty) \times D \rightarrow \mathbb{R}$ satisfies locally Lipschitz condition on $x ; D \subset \mathbb{R}^{m}$ is a domain containing origin; $t \geq 0, \beta \in(0,1), \alpha_{1}, \alpha_{2}, \alpha_{3}, a$ and $b$ are arbitrary positive constants. If the assumptions hold globally on $\mathbb{R}^{m}$, then $x^{*}=0$ is globally Mittag-Leffler stable.

Theorem 2.10. (The Extended Second Method of Lyapunov [14]) For $t_{0}=0$, the fractional-order system (2.8) is Mittag-Leffler stable at the equilibrium point $x^{*}=0$, if there exists a continuous function $V(t, x(t))$ satisfying

$$
\begin{aligned}
\alpha_{1}\|x(t)\|^{a} & \leq V(t, x(t)) \leq \alpha_{2}\|x(t)\|^{a b}, \\
D_{t}^{\beta} V\left(t^{+}, x\left(t^{+}\right)\right) & \leq-\alpha_{3}\|x\|^{a b}, \text { (holding almost everywhere) }
\end{aligned}
$$

where $V(t, x(t)):[0, \infty) \times D \rightarrow \mathbb{R}$ satisfies locally Lipschitz condition on $x ; \dot{V}(t, x(t))$ is piecewise continuous, and $\lim _{s \rightarrow t^{+}} \dot{V}(s, x(s))$ exists for any $t \in[0, \infty) ; D \subset \mathbb{R}^{m}$ is a domain containing origin and $V\left(t^{+}, x\left(t^{+}\right)\right)=\lim _{s \rightarrow t^{+}} V(s, x(s)) ; t \geq 0, \beta \in(0,1), \alpha_{1}, \alpha_{2}, \alpha_{3}, a$ and $b$ are arbitrary positive constants. If the assumptions hold globally on $\mathbb{R}^{m}$, then $x^{*}=0$ is globally Mittag-Leffler stable.

Theorem 2.11. [14] If $h(t) \in C^{1}([0,+\infty), \mathbb{R})$, then the following inequality holds almost everywhere.

$$
D_{t}^{\alpha}\left|h\left(t^{+}\right)\right| \leq \operatorname{sgn}(h(t)) D_{t}^{\alpha} h(t), 0<\alpha<1,
$$

where $h\left(t^{+}\right)=\lim _{s \rightarrow t^{+}} h(s)$, where $s g n$ denotes the signum function. 
Lemma 2.12. [20] Let $m$ be a positive integer such that $m-1<\alpha<m$; if $x(t) \in C^{m-1}[a, b]$, then

$$
I_{t}^{\alpha} D_{t}^{\alpha} x(t)=x(t)-\sum_{k=0}^{m-1} \frac{x^{k}(a)}{k !}(t-a)^{k} .
$$

In particular, if $0<\alpha \leq 1$ and $x(t) \in C[a, b]$, then we have the Fundamental Theorem of Calculus

$$
I_{t}^{\alpha} D_{t}^{\alpha} x(t)=x(t)-x(a) .
$$

Lemma 2.13. [33] Suppose that $\alpha>0, x(t)$ be a non-negative function locally integrable on $0 \leq t<T$ (for some $T \leq+\infty$ ) and $v$ be a non-negative, non-decreasing continuous function defined on $0 \leq t<T, v(t) \leq M$ (real constant). Furthermore, if $u(t)$ is non-negative and locally integrable on $0 \leq t<T$ such that

$$
x(t) \leq u(t)+v(t) \int_{0}^{t}(t-s)^{\alpha-1} x(s) d s,
$$

on $0 \leq t<T$. Then

$$
x(t) \leq u(t)+\int_{0}^{t}\left[\sum_{m=1}^{\infty} \frac{(v(t) \Gamma(\alpha))^{m}}{\Gamma(n \alpha)}(t-s)^{m \alpha-1} u(s)\right] d s, t \in[0, T) .
$$

Henry [18] obtained (2.16) for $u$ and $v$ constant. For the use of (2.16), see [19].

Remark 2.14. If in Lemma 2.13, $u(t)$ is a non-decreasing function on $0 \leq t<T$, then

$$
x(t) \leq u(t) E_{\alpha, 1}\left(v(t) \Gamma(\alpha) t^{\alpha}\right), t \in[0, T) .
$$

\section{Global Mittag-Leffler stability}

In this section, we obtain sufficient condition for global Mittag-Leffler stability of the system (1.1), which further implies the global asymptotic stability of the equilibrium point.

We introduce the following conditions on our model system (1.1).

A1. The activation functions $f($.$) and g($.$) are continuous and Lipschitz, i.e. for some x, y \in \mathbb{R}$, there exist positive constants $L_{f}$ and $L_{g}$ such that

$$
\begin{aligned}
|f(x)-f(y)| & \leq L_{f}|x-y|, \\
|g(x)-g(y)| & \leq L_{g}|x-y| .
\end{aligned}
$$

A2. There exists positive constant $c_{i}(i=1,2, \cdots, m)$ such that

$$
c_{i}=\frac{1}{a_{i}}\left(\sum_{j=1}^{m}\left|b_{j i}\right| L_{f}+\sum_{j=1}^{m}\left|c_{j i}\right| L_{g}\right)<1 .
$$


A3. The system parameters $a_{i}, b_{i j}, c_{i j}(i, j=1,2, \cdots, m)$ satisfy

$$
\min _{1 \leq i \leq m}\left(a_{i}-L_{f} \sum_{j=1}^{m}\left|b_{j i}\right|\right)>\max _{1 \leq i \leq m}\left(L_{g} \sum_{j=1}^{m}\left|c_{j i}\right|\right)>0 .
$$

A4. The time delay term $\tau$ is a positive real number.

Remark 3.1. The problems of existence and uniqueness of equilibrium solutions of fractional order delayed neural networks have been studied by several authors in literature. For more details, refer to [20-22]. For convenience of reader, we give the proof of existence and uniqueness of equilibrium point of system (1.1) as following theorem.

Theorem 3.2. If the Assumptions (A1)-(A2) hold, then there exists a unique equilibrium point of system (1.1).

Proof. Define $\|x\|=\|x\|_{1}$, that is $\|x\|=\sum_{i=1}^{m}\left|x_{i}\right|$ for any $x=\left(x_{1}, x_{2}, \cdots, x_{m}\right)^{T} \in \mathbb{R}^{m}$. Consider the mapping $F(y)=\left(F_{1}\left(y_{1}\right), F_{2}\left(y_{2}\right), \cdots, F_{m}\left(y_{m}\right)\right)^{T}$, where $y=\left(y_{1}, y_{2}, \cdots, y_{m}\right)^{T} \in \mathbb{R}^{m}$ and

$$
F_{i}\left(y_{i}\right)=\sum_{j=1}^{m} b_{i j} f\left(\frac{y_{j}}{a_{j}}\right)+\sum_{j=1}^{m} c_{i j} g\left(\frac{y_{j}}{a_{j}}\right)+I_{i}, \quad(i=1,2, \cdots, m) .
$$

We will show that $F$ is a contraction mapping on $\mathbb{R}^{m}$. From Assumption (A1), for any two vectors $y, z \in \mathbb{R}^{m}$, we have

$$
\begin{aligned}
\left|F_{i}(y)-F_{i}(z)\right| & =\left|\sum_{j=1}^{m} b_{i j}\left(f\left(\frac{y_{j}}{a_{j}}\right)-f\left(\frac{z_{j}}{a_{j}}\right)\right)\right|+\left|\sum_{j=1}^{m} c_{i j}\left(g\left(\frac{y_{j}}{a_{j}}\right)-g\left(\frac{z_{j}}{a_{j}}\right)\right)\right| \\
& \leq\left(\sum_{j=1}^{m} \frac{\left|b_{i j}\right| L_{f}}{a_{j}}+\sum_{j=1}^{m} \frac{\left|c_{i j}\right| L_{g}}{a_{j}}\right)\left|y_{j}-z_{j}\right|
\end{aligned}
$$

From Assumption (A2), we obtain

$$
\begin{aligned}
\|F(y)-F(z)\| & =\sum_{i=1}^{m}\left|F_{i}(y)-F_{i}(z)\right| \\
& \leq \sum_{i=1}^{m} \sum_{j=1}^{m} \frac{1}{a_{j}}\left(\left|b_{i j}\right| L_{f}+\left|c_{i j}\right| L_{g}\right)\left|y_{j}-z_{j}\right| \\
& =\sum_{i=1}^{m}\left(\sum_{j=1}^{m} \frac{1}{a_{i}}\left(\left|b_{j i}\right| L_{f}+\left|c_{j i}\right| L_{g}\right)\right)\left|y_{i}-z_{i}\right| \\
& <c\|y-z\|,
\end{aligned}
$$


where $c=\max \left\{c_{1}, c_{2}, \cdots, c_{m}\right\}$. Thus we have $\|F(y)-F(z)\|<c\|y-z\|$. Hence the mapping $F: \mathbb{R}^{m} \rightarrow \mathbb{R}^{m}$ is a strict contraction mapping on $\mathbb{R}^{m}$ with Lipschitz constant $c$. Thus there exists a unique fixed point $y^{*} \in \mathbb{R}^{m}$ such that $F\left(y^{*}\right)=y^{*}$, that is

$$
y_{i}^{*}=\sum_{j=1}^{m} b_{i j} f\left(\frac{y_{j}^{*}}{a_{j}}\right)+\sum_{j=1}^{m} c_{i j} g\left(\frac{y_{j}^{*}}{a_{j}}\right)+I_{i}, \quad(i=1,2, \cdots, m) .
$$

Denote $x_{i}^{*}=\frac{y_{i}^{*}}{a_{i}}$ for $i=1,2, \cdots, m$. Then we have

$$
-a_{i} x_{i}^{*}+\sum_{j=1}^{m} b_{i j} f\left(x_{j}^{*}\right)+\sum_{j=1}^{m} c_{i j} g\left(x_{j}^{*}\right)+I_{i}=0, \quad(i=1,2, \cdots, m) .
$$

Thus $x^{*}=\left(x_{1}^{*}, x_{2}^{*}, \cdots, x_{m}^{*}\right)^{T}$ is the unique equilibrium point of system (1.1).

Q.E.D.

Now we prove the Mittag-Leffler stability of the unique equilibrium point of system (1.1).

Let $x^{*}=\left(x_{1}^{*}, x_{2}^{*}, \cdots, x_{m}^{*}\right)^{T}$ be the unique equilibrium point of (1.1). Then for $e \in \mathbb{R}^{m}, e=$ $\left(e_{1}, e_{2}, \cdots, e_{m}\right)^{T}$, it can be derived from (1.1) that the error $e_{i}(t)=x_{i}(t)-x_{i}^{*}$ satisfies

$$
\begin{aligned}
D_{t}^{\alpha} e_{i}(t) & =-a_{i} e_{i}(t)+\sum_{j=1}^{m} b_{i j}\left(f\left(x_{j}^{*}+e_{j}(t)\right)-f\left(x_{j}^{*}\right)\right) \\
& +\sum_{j=1}^{m} c_{i j}\left(g\left(x_{j}^{*}+e_{j}(t-\tau)\right)-g\left(x_{j}^{*}\right)\right) \\
e_{i}(t) & =\varphi_{i}(t)-x_{i}^{*}, \quad t \in[-\tau, 0] \quad i=1,2, \cdots, m .
\end{aligned}
$$

Theorem 3.3. If Assumptions $(A 1)-(A 4)$ hold, then the equilibrium point $x^{*}=\left(x_{1}^{*}, x_{2}^{*}, \cdots, x_{m}^{*}\right)^{T}$ of system (1.1) is globally Mittag-Leffler stable.

Proof. We construct a Lyapunov function as

$$
V(t, e(t))=\|e(t)\|=\sum_{i=1}^{m}\left|e_{i}(t)\right|
$$

It can be observed that the Lyapunov function satisfies equation (2.9) of Theorem 2.10. From 
Assumptions $(A 1),(A 3)$ and Theorem 2.11, the following inequality holds almost everywhere;

$$
\begin{aligned}
D_{t}^{\alpha} V\left(t^{+}, e\left(t^{+}\right)\right) & =\sum_{i=1}^{m} D_{t}^{\alpha}\left|e_{i}\left(t^{+}\right)\right| \leq \sum_{i=1}^{m} \operatorname{sgn}\left(e_{i}(t)\right) D_{t}^{\alpha} e_{i}(t) \\
& \leq \sum_{i=1}^{m}\left[-a_{i}\left|e_{i}(t)\right|+\sum_{j=1}^{m} L_{f}\left|b_{i j}\right|\left|e_{j}(t)\right|+\sum_{j=1}^{m} L_{g}\left|c_{i j}\right|\left|e_{j}(t-\tau)\right|\right] \\
& =-\sum_{i=1}^{m}\left[a_{i}-\sum_{j=1}^{m} L_{f}\left|b_{j i}\right|\right]\left|e_{i}(t)\right|+\sum_{j=1}^{m} \sum_{i=1}^{m} L_{g}\left|c_{i j}\right|\left|e_{j}(t-\tau)\right| \\
& \leq-k_{1} V(t, e(t))+k_{2} \sup _{t-\tau \leq s \leq t} V(s, e(s)),
\end{aligned}
$$

where

$$
k_{1}=\min _{1 \leq i \leq m}\left(a_{i}-L_{f} \sum_{j=1}^{m}\left|b_{j i}\right|\right)>0, \text { and } k_{2}=\max _{1 \leq i \leq m}\left(L_{g} \sum_{j=1}^{m}\left|c_{j i}\right|\right)>0 .
$$

Thus from above estimate, for any solution $e(t)$ of (3.3), that satisfies the Razumikhin condition

$$
V(s, e(s)) \leq V(t, e(t)), \quad t-\tau \leq s \leq t,
$$

we have

$$
D_{t}^{\alpha} V\left(t^{+}, e\left(t^{+}\right)\right) \leq-\left(k_{1}-k_{2}\right) V(t, e(t)) .
$$

Form Assumption $(A 3)$, there exists a real number $\gamma>0$, such that

$$
k_{1}-k_{2} \geq \gamma
$$

Thus it follows that

$$
D_{t}^{\alpha} V\left(t^{+}, e\left(t^{+}\right)\right) \leq-\gamma V(t, e(t)) .
$$

It can be observed that the condition (2.12) of Theorem 2.10 is satisfied. Thus, we have

$$
\|e(t)\|=V(t, e(t)) \leq V(0, e(0)) E_{\alpha}\left(-\gamma t^{\alpha}\right) .
$$

Hence,

$$
\begin{aligned}
\left\|x(t)-x^{*}\right\|=\sum_{i=1}^{m}\left|x_{i}(t)-x_{i}^{*}\right| & \leq E_{\alpha}\left(-\gamma t^{\alpha}\right) \sum_{i=1}^{m}\left|x_{i}(0)-x_{i}^{*}\right| \\
& =\left\|\varphi_{i}(0)-x^{*}\right\| E_{\alpha}\left(-\gamma t^{\alpha}\right) \\
& \leq\left\|\varphi_{0}-x^{*}\right\|_{\tau} E_{\alpha}\left(-\gamma t^{\alpha}\right) .
\end{aligned}
$$

Let $m=\left\|\varphi_{0}-x^{*}\right\|_{\tau}$, where $m \geq 0$ and $m=0$ holds only if $\varphi_{i}(0)=x^{*},(i=1,2, \cdots, m)$. Then $\left\|x(t)-x^{*}\right\| \leq m E_{\alpha}\left(-\gamma t^{\alpha}\right)$. Thus system (1.1) is globally Mittag-Leffler stable.

Q.E.D.

Remark 3.4. Since the equilibrium point $x^{*}$ of system (1.1) is globally Mittag-Leffler stable. Thus from Remark 2.8 , it is globally asymptotically stable. 


\section{Approximation Results}

\subsection{Formulation of discrete-time analogue: Euler's polygonal lines of order $\alpha$}

Recently, many researchers have applied the discretization method to discretize various cellular neural networks [23-29]. But discretizing the fractional-order differential equations is of main interest. In [30], Riccati's fractional-order differential equation is discretized. It has been observed that as the fractional-order parameter $\alpha \rightarrow 1$, Euler's discretization method is obtained. In [31], logistic fractional-order differential equation is discretized using same method. In [32], discretization method is applied to Chua's system, which is one of the autonomous differential equations capable of generating chaotic behavior. Fixed points and their asymptotic stability are also investigated. Chaotic attractor, bifurcation and chaos for different values of the fractional-order parameter have been discussed. In the next result, we propose a numerical discretization scheme using piecewise constant arguments of generalized type, which has not been derived till now to the best of author's knowledge.

Let $0<\alpha \leq 1$, and consider the following fractional-order delayed neural network.

$$
\begin{aligned}
D_{t}^{\alpha} x_{i}(t) & =-a_{i} x_{i}(t)+\sum_{j=1}^{m} b_{i j} f\left(x_{j}(t)\right)+\sum_{j=1}^{m} c_{i j} g\left(x_{j}(t-\tau)\right)+I_{i} \quad t \in[0, T) \\
x_{i}(t) & =\varphi_{i}(t), \quad t \in[-\tau, 0], \quad T<\infty, \quad i=1,2, \cdots, m .
\end{aligned}
$$

Let $\gamma_{h}(t)=h\left[\frac{t}{h}\right]$, where [.] is the usual greatest integer function and $h$ is a positive number in the interval $(0, \tau]$. In fact we can consider $h=\frac{\tau}{\nu}$ with $\nu \geq 1$ is an integer. It is clear that $\gamma_{h}(t)=h n$ for $h n \leq t \leq h(n+1)$, and $n$ is a positive integer, also we have $\gamma_{h}(\tau)=\nu h$.

The corresponding differential equation with piecewise constant arguments $\gamma_{h}(t)=h\left[\frac{t}{h}\right]$ is given by

$$
\begin{aligned}
D_{t}^{\alpha} z_{i}^{h}(t)= & -a_{i} z_{i}^{h}\left(\gamma_{h}(t)\right)+\sum_{j=1}^{m} b_{i j} f\left(z_{j}^{h}\left(\gamma_{h}(t)\right)\right) \\
& +\sum_{j=1}^{m} c_{i j} g\left(z_{j}^{h}\left(\gamma_{h}(t-\tau)\right)\right)+I_{i},
\end{aligned}
$$

with initial conditions

$$
z_{i}^{h}(n h)=\varphi_{i}(n h), \quad n=-\nu, \cdots, 0 .
$$

By the solution of above system (4.2)-(4.3), we mean a function $z^{h}=\left(z_{i}^{h}\right), i=1,2, \cdots, m$ defined on $\{-j h: j=0,1, \cdots$, such that $-\tau \leq-j h \leq 0\}$ by (4.3), which satisfy the following properties on $\mathbb{R}^{+}$;

1. The functions $z_{i}^{h}$ are continuous for each $i=1,2, \cdots, m$ on $\mathbb{R}^{+}$. 
2. The corresponding derivatives of $z_{i}^{h}(t)$ exist at each point $t \in \mathbb{R}^{+}$with the possible exception of the points $j h(j=0,1, \cdots)$ where finite one-sided derivatives exists, for each $i=1,2, \cdots, m$.

3. The function $z_{i}^{h}$ satisfies (4.2) on each interval $I_{(j, h)}=[j h,(j+1) h)$ for $j=0,1, \cdots$.

On $I_{(n, h)}$ we can rewrite equation (4.2)-(4.3) as

$$
D_{t}^{\alpha} z_{i}^{h}(t)=-a_{i} z_{i}^{h}(n h)+\sum_{j=1}^{m} b_{i j} f\left(z_{j}^{h}(n h)\right)+\sum_{j=1}^{m} c_{i j} g\left(z_{j}^{h}(h(n-\nu))\right)+I_{i} .
$$

As $\gamma_{h}$ has been applied also in the linear part, the discretization method obtained is the $\alpha$-fractional polygonal of Euler [30]. Our discretization method proceed as follows:

1. Let $t \in[0, h)$, then $\frac{t}{h} \in[0,1)$. Thus we get, for $t \in[0, h)$

$$
D_{t}^{\alpha} z_{i}^{h}(t)=-a_{i} z_{i}^{h}(0)+\sum_{j=1}^{m} b_{i j} f\left(z_{j}^{h}(0)\right)+\sum_{j=1}^{m} c_{i j} g\left(z_{j}^{h}(h(0-\nu))\right)+I_{i},
$$

and by the Fundamental Theorem of Fractional Calculus (2.15), the solution of (4.4) is given by

$$
\begin{aligned}
z_{i}^{h}(t)= & z_{i}^{h}(0)+\frac{1}{\Gamma \alpha} \int_{0}^{t}(t-s)^{\alpha-1}\left(-a_{i} z_{i}^{h}(0)+\sum_{j=1}^{m} b_{i j} f\left(z_{j}^{h}(0)\right)\right. \\
& \left.+\sum_{j=1}^{m} c_{i j} g\left(z_{j}^{h}(h(0-\nu))\right)+I_{i}\right) d s
\end{aligned}
$$

or

$$
\begin{aligned}
z_{i}^{h}(t)= & z_{i}^{h}(0)+\frac{t^{\alpha}}{\Gamma(\alpha+1)}\left(-a_{i} z_{i}^{h}(0)+\sum_{j=1}^{m} b_{i j} f\left(z_{j}^{h}(0)\right)\right. \\
& \left.+\sum_{j=1}^{m} c_{i j} g\left(z_{j}^{h}(h(0-\nu))\right)+I_{i}\right)
\end{aligned}
$$

and by the continuity of $z_{i}^{h}(t)$ at $t=h$, we obtain

$$
\begin{aligned}
z_{i}^{h}(h)= & \left(1-a_{i} \frac{h^{\alpha}}{\Gamma(\alpha+1)}\right) z_{i}^{h}(0) \\
& +\frac{h^{\alpha}}{\Gamma(\alpha+1)}\left(\sum_{j=1}^{m} b_{i j} f\left(z_{j}^{h}(0)\right)+\sum_{j=1}^{m} c_{i j} g\left(z_{j}^{h}(h(0-\nu))\right)+I_{i}\right) .
\end{aligned}
$$


2. Let $t \in[h, 2 h)$, then $\frac{t}{h} \in[1,2)$. Then we have

$$
D_{t}^{\alpha} z_{i}^{h}(t)=-a_{i} z_{i}^{h}(h)+\sum_{j=1}^{m} b_{i j} f\left(z_{j}^{h}(h)\right)+\sum_{j=1}^{m} c_{i j} g\left(z_{j}^{h}(h(1-\nu))\right)+I_{i} .
$$

The solution on $[h, 2 h)$ of above is given as

$$
\begin{aligned}
z_{i}^{h}(t)= & z_{i}^{h}(h)+\frac{1}{\Gamma \alpha} \int_{h}^{t}(t-s)^{\alpha-1}\left(-a_{i} z_{i}^{h}(h)+\sum_{j=1}^{m} b_{i j} f\left(z_{j}^{h}(h)\right)\right. \\
& \left.+\sum_{j=1}^{m} c_{i j} g\left(z_{j}^{h}(h(1-\nu))\right)+I_{i}\right) d s,
\end{aligned}
$$

or

$$
\begin{aligned}
z_{i}^{h}(t)= & z_{i}^{h}(h)-a_{i} \frac{(t-h)^{\alpha}}{\Gamma(\alpha+1)} z_{i}^{h}(h) \\
& +\frac{(t-h)^{\alpha}}{\Gamma(\alpha+1)}\left(\sum_{j=1}^{m} b_{i j} f\left(z_{j}^{h}(h)\right)+\sum_{j=1}^{m} c_{i j} g\left(z_{j}^{h}(h(1-\nu))\right)+I_{i}\right),
\end{aligned}
$$

and by the continuity of $z_{i}^{h}(t)$ at $t=2 h$, we obtain

$$
\begin{aligned}
z_{i}^{h}(2 h)= & \left(1-a_{i} \frac{h^{\alpha}}{\Gamma(\alpha+1)}\right) z_{i}^{h}(h) \\
& +\frac{h^{\alpha}}{\Gamma(\alpha+1)}\left(\sum_{j=1}^{m} b_{i j} f\left(z_{j}^{h}(h)\right)+\sum_{j=1}^{m} c_{i j} g\left(z_{j}^{h}(h(1-\nu))\right)+I_{i}\right) .
\end{aligned}
$$

Using (4.5), we can rewrite (4.6) as

$$
\begin{aligned}
z_{i}^{h}(2 h)= & \left(1-a_{i} \frac{h^{\alpha}}{\Gamma(\alpha+1)}\right)\left(1-a_{i} \frac{h^{\alpha}}{\Gamma(\alpha+1)}\right) z_{i}^{h}(0) \\
& +\left(1-a_{i} \frac{h^{\alpha}}{\Gamma(\alpha+1)}\right) \frac{h^{\alpha}}{\Gamma(\alpha+1)}\left(\sum_{j=1}^{m} b_{i j} f\left(z_{j}^{h}(0)\right)\right. \\
& \left.+\sum_{j=1}^{m} c_{i j} g\left(z_{j}^{h}(h(0-\nu))\right)+I_{i}\right) \\
& +\frac{h^{\alpha}}{\Gamma(\alpha+1)}\left(\sum_{j=1}^{m} b_{i j} f\left(z_{j}^{h}(h)\right)+\sum_{j=1}^{m} c_{i j} g\left(z_{j}^{h}(h(1-\nu))\right)+I_{i}\right) .
\end{aligned}
$$

Repeating the process, it can be easily deduced that for $n h \leq t<(n+1) h$, the solution of IVP 
(4.4) can be given as

$$
\begin{aligned}
z_{i}^{h}(t)= & z_{i}^{h}(n h)-a_{i} \frac{(t-n h)^{\alpha}}{\Gamma(\alpha+1)} z_{i}^{h}(n h) \\
& +\frac{(t-n h)^{\alpha}}{\Gamma(\alpha+1)}\left(\sum_{j=1}^{m} b_{i j} f\left(z_{j}^{h}(n h)\right)+\sum_{j=1}^{m} c_{i j} g\left(z_{j}^{h}(h(n-\nu))\right)+I_{i}\right) .
\end{aligned}
$$

Replacing $t=(n+1) h$ in (4.7) and using continuity of $z_{i}^{h}$, we obtain

$$
\begin{aligned}
z_{i}^{h}((n+1) h)= & \left(1-a_{i} \frac{h^{\alpha}}{\Gamma(\alpha+1)}\right) z_{i}^{h}(n h) \\
& +\frac{h^{\alpha}}{\Gamma(\alpha+1)}\left(\sum_{j=1}^{m} b_{i j} f\left(z_{j}^{h}(n h)\right)+\sum_{j=1}^{m} c_{i j} g\left(z_{j}^{h}(h(n-\nu))\right)+I_{i}\right) .
\end{aligned}
$$

Define sequence $\mathfrak{a}_{i}^{h}(n)=z_{i}^{h}(n h)$. This sequence satisfies the following non-linear system of delay difference equations

$$
\begin{aligned}
\mathfrak{a}_{i}^{h}(n+1)= & \left(1-a_{i} \frac{h^{\alpha}}{\Gamma(\alpha+1)}\right) \mathfrak{a}_{i}^{h}(n) \\
& +\frac{h^{\alpha}}{\Gamma(\alpha+1)}\left(\sum_{j=1}^{m} b_{i j} f\left(\mathfrak{a}_{j}^{h}(n)\right)+\sum_{j=1}^{m} c_{i j} g\left(\mathfrak{a}_{j}^{h}(n-\nu)\right)+I_{i}\right)
\end{aligned}
$$

with initial condition

$$
\mathfrak{a}_{i}^{h}(n)=\varphi_{i}(n h) \quad n=-\nu, \cdots, 0 .
$$

The initial value problem (4.9)-(4.10) is a discretization of the original problem (4.1). 
From the above recurrence relation and initial conditions, we obtain

$$
\begin{aligned}
\mathfrak{a}_{i}^{h}(0)= & \varphi_{i}(0) \\
\mathfrak{a}_{i}^{h}(1)= & \left(1-a_{i} \frac{h^{\alpha}}{\Gamma(\alpha+1)}\right) \mathfrak{a}_{i}^{h}(0) \\
& +\frac{h^{\alpha}}{\Gamma(\alpha+1)}\left(\sum_{j=1}^{m} b_{i j} f\left(\mathfrak{a}_{j}^{h}(0)\right)+\sum_{j=1}^{m} c_{i j} g\left(\mathfrak{a}_{j}^{h}(0-\nu)\right)+I_{i}\right) \\
= & \left(1-a_{i} \frac{h^{\alpha}}{\Gamma(\alpha+1)}\right) \varphi_{i}(0) \\
& +\frac{h^{\alpha}}{\Gamma(\alpha+1)}\left(\sum_{j=1}^{m} b_{i j} f\left(\varphi_{j}(0)\right)+\sum_{j=1}^{m} c_{i j} g\left(\varphi_{j}(0-\nu)\right)+I_{i}\right) \\
\mathfrak{a}_{i}^{h}(2)= & \left(1-a_{i} \frac{h^{\alpha}}{\Gamma(\alpha+1)}\right) \mathfrak{a}_{i}^{h}(1) \\
& +\frac{h^{\alpha}}{\Gamma(\alpha+1)}\left(\sum_{j=1}^{m} b_{i j} f\left(\mathfrak{a}_{j}^{h}(1)\right)+\sum_{j=1}^{m} c_{i j} g\left(\mathfrak{a}_{j}^{h}(1-\nu)\right)+I_{i}\right) \\
& +\frac{h^{\alpha}}{\Gamma(\alpha+1)}\left(\sum_{j=1}^{m} b_{i j} f\left(\mathfrak{a}_{j}^{h}(1)\right)+\sum_{j=1}^{m} c_{i j} g\left(\varphi_{j}(1-\nu)\right)+I_{i}\right) \\
& +\left(1-a_{i} \frac{h^{\alpha}}{\Gamma(\alpha+1)}\right)\left(1-a_{i} \frac{h^{\alpha}}{\Gamma(\alpha+1)}\right) \varphi_{i}(0) \\
& \left(h^{\alpha}\right) \frac{h^{\alpha}}{\Gamma(\alpha+1)}\left(\sum_{j=1}^{m} b_{i j} f\left(\varphi_{j}(0)\right)+\sum_{j=1}^{m} c_{i j} g\left(\varphi_{j}(0-\nu)\right)+I_{i}\right)
\end{aligned}
$$

Based on the above calculations, we can deduce that

$$
\begin{aligned}
\mathfrak{a}_{i}^{h}(n+1)= & \left(1-a_{i} \frac{h^{\alpha}}{\Gamma(\alpha+1)}\right)^{(n+1)} \varphi_{i}(0) \\
& +\sum_{k=0}^{n}\left(1-a_{i} \frac{h^{\alpha}}{\Gamma(\alpha+1)}\right)^{(n-k)} \frac{h^{\alpha}}{\Gamma(\alpha+1)}\left[\sum_{j=1}^{m} b_{i j} f\left(\mathfrak{a}_{j}^{h}(k)\right)\right. \\
& \left.+\sum_{j=1}^{m} c_{i j} g\left(\mathfrak{a}_{j}^{h}(k-\nu)\right)+I_{i}\right]
\end{aligned}
$$

From the initial conditions, the above sequence $\mathfrak{a}_{i}^{h}(n)$ is well defined. Hence the solution of system 
(4.3), for $t \in I_{(n, h)}$, can be written as

$$
\begin{aligned}
z_{i}^{h}(t)= & \mathfrak{a}_{i}^{h}(n)+\frac{1}{\Gamma \alpha} \int_{n h}^{t}(t-s)^{\alpha-1}\left(-a_{i} \mathfrak{a}_{i}^{h}(n)+\sum_{j=1}^{m} b_{i j} f\left(\mathfrak{a}_{j}^{h}(n)\right)\right. \\
& \left.+\sum_{j=1}^{m} c_{i j} g\left(\mathfrak{a}_{j}^{h}(n-\nu)\right)+I_{i}\right) d s
\end{aligned}
$$

are the $\alpha$-fractional Euler method. We can write the above result as the following theorem.

Theorem 4.1. The system (4.2)-(4.3) has a unique solution, for $t \in I_{(n, h)}$, in the following form:

$$
\begin{aligned}
z_{i}^{h}(t)= & {\left[1-a_{i}\left(\frac{(t-n h)^{\alpha}}{\Gamma(\alpha+1)}\right)\right] \mathfrak{a}_{i}^{h}(n) } \\
& +\left(\frac{(t-n h)^{\alpha}}{\Gamma(\alpha+1)}\right)\left(\sum_{j=1}^{m} b_{i j} f\left(\mathfrak{a}_{j}^{h}(n)\right)+\sum_{j=1}^{m} c_{i j} g\left(\mathfrak{a}_{j}^{h}(n-\nu)\right)+I_{i}\right) .
\end{aligned}
$$

for $t \geq 0, i=1,2, \cdots, m$ and the sequence $\mathfrak{a}_{i}^{h}($.$) satisfies the non linear difference equation (4.9)$ with the initial conditions given by (4.10).

Note 4.2. The approximation method with piecewise constant argument described above is valid only if the solutions of system of non-linear delayed differential equations (4.1), (4.3) and (4.9)-(4.10) are unique.

\subsection{Approximation Results in Bounded Intervals: Error function}

In this section, it is proved that system (4.1) can be approximated for $t \in[0, T)$ by the Euler schema.

To prove the result, we use the particular form of Lemma 2.12, i.e. for $0<\alpha \leq 1$ and $x(t) \in C[a, b]$,

$$
I_{t}^{\alpha} D_{t}^{\alpha} x(t)=x(t)-x(a) .
$$

Let there exists a positive constant $r$. Then for every $h \in(0, r)$ and $t \in I_{(n, h)}=[n h,(n+1) h), n \geq 0$, we define

$$
\varepsilon_{i}^{h}(t):=x_{i}(t)-z_{i}^{h}(t) .
$$

We can rewrite (4.18) as

$$
\begin{aligned}
\varepsilon_{i}^{h}(t) & =x_{i}(t)-z_{i}^{h}(t)-\varphi_{i}(0)+\varphi_{i}(0) \\
& =\left[x_{i}(t)-\varphi_{i}(0)\right]-\left[z_{i}^{h}(t)-\varphi_{i}(0)\right] \\
& =I_{t}^{\alpha} D_{t}^{\alpha} x_{i}(t)-I_{t}^{\alpha} D_{t}^{\alpha} z_{i}^{h}(t) .
\end{aligned}
$$

Since from Definition (2.1), we have

$$
I_{t}^{\alpha} f(t)=\frac{1}{\Gamma \alpha} \int_{0}^{t}(t-s)^{\alpha-1} f(s) d s .
$$


Using (4.20) in equation (4.19), we obtain

$$
\begin{aligned}
\varepsilon_{i}^{h}(t)= & \frac{1}{\Gamma \alpha} \int_{0}^{t}(t-s)^{\alpha-1} D_{t}^{\alpha}\left[x_{i}(s)-z_{i}^{h}(s)\right] d s \\
= & \frac{-a_{i}}{\Gamma \alpha} \int_{0}^{t}(t-s)^{\alpha-1}\left[x_{i}(s)-z_{i}^{h}\left(\gamma_{h}(s)\right)\right] d s \\
& +\frac{1}{\Gamma \alpha} \int_{0}^{t}(t-s)^{\alpha-1} \sum_{j=1}^{m} b_{i j}\left[f\left(x_{j}(s)\right)-f\left(z_{j}^{h}\left(\gamma_{h}(s)\right)\right)\right] d s \\
& +\frac{1}{\Gamma \alpha} \int_{0}^{t}(t-s)^{\alpha-1} \sum_{j=1}^{m} c_{i j}\left[g\left(x_{j}(s-\tau)\right)-g\left(z_{j}^{h}\left(\gamma_{h}(s-\tau)\right)\right)\right] d s .
\end{aligned}
$$

Note that

$$
\left|x_{i}(s)-z_{i}^{h}\left(\gamma_{h}(s)\right)\right| \leq\left|x_{i}(s)-x_{i}\left(\gamma_{h}(s)\right)\right|+\left|x_{i}\left(\gamma_{h}(s)\right)-z_{i}^{h}\left(\gamma_{h}(s)\right)\right| .
$$

Using Lipschitz continuity of $f($.$) and g($.$) , we have$

$$
\begin{aligned}
\left|\varepsilon_{i}^{h}(t)\right| \leq & \frac{a_{i}}{\Gamma \alpha} \int_{0}^{t}(t-s)^{\alpha-1}\left|\varepsilon_{i}^{h}\left(\gamma_{h}(s)\right)\right| d s \\
& +\frac{a_{i}}{\Gamma \alpha} \int_{0}^{t}(t-s)^{\alpha-1}\left|x_{i}(s)-x_{i}\left(\gamma_{h}(s)\right)\right| d s \\
& +\frac{L_{f}}{\Gamma \alpha} \int_{0}^{t}(t-s)^{\alpha-1} \sum_{j=1}^{m}\left|b_{i j}\right|\left|\varepsilon_{j}^{h}\left(\gamma_{h}(s)\right)\right| d s \\
& +\frac{L_{f}}{\Gamma \alpha} \int_{0}^{t}(t-s)^{\alpha-1} \sum_{j=1}^{m}\left|b_{i j}\right|\left|x_{i}(s)-x_{i}\left(\gamma_{h}(s)\right)\right| d s \\
& +L_{g} \frac{1}{\Gamma \alpha} \int_{0}^{t}(t-s)^{\alpha-1} \sum_{j=1}^{m}\left|c_{i j}\right|\left|\varepsilon_{j}^{h}\left(\gamma_{h}(s-\tau)\right)\right| d s \\
& +L_{g} \frac{1}{\Gamma \alpha} \int_{0}^{t}(t-s)^{\alpha-1} \sum_{j=1}^{m}\left|c_{i j}\right|\left|x_{j}(s-\tau)-x_{j}\left(\gamma_{h}(s-\tau)\right)\right| d s
\end{aligned}
$$


Now we take the sum of the above inequalities from 1 to $m$, and obtain

$$
\begin{aligned}
& \sum_{i=1}^{m}\left|\varepsilon_{i}^{h}(t)\right| \\
& \leq \sum_{i=1}^{m} \frac{a_{i}}{\Gamma \alpha} \int_{0}^{t}(t-s)^{\alpha-1}\left(\sum_{i=1}^{m}\left|\varepsilon_{i}^{h}\left(\gamma_{h}(s)\right)\right|\right) d s \\
& +\frac{L_{f}}{\Gamma \alpha} \int_{0}^{t}(t-s)^{\alpha-1} \sum_{1 \leq i, j \leq m}\left|b_{i j}\right|\left(\sum_{i=1}^{m}\left|\varepsilon_{i}^{h}\left(\gamma_{h}(s)\right)\right|\right) d s \\
& +\frac{L_{g}}{\Gamma \alpha} \int_{0}^{t}(t-s)^{\alpha-1} \sum_{1 \leq i, j \leq m}\left|c_{i j}\right|\left(\sum_{i=1}^{m}\left|\varepsilon_{i}^{h}\left(\gamma_{h}(s-\tau)\right)\right|\right) d s \\
& +\sum_{i=1}^{m} \frac{a_{i}}{\Gamma \alpha} \int_{0}^{t}(t-s)^{\alpha-1}\left(\sum_{i=1}^{m}\left|x_{i}(s)-x_{i}\left(\gamma_{h}(s)\right)\right|\right) d s \\
& +\frac{L_{f}}{\Gamma \alpha} \int_{0}^{t}(t-s)^{\alpha-1} \sum_{1 \leq i, j \leq m}\left|b_{i j}\right|\left(\sum_{i=1}^{m}\left|x_{i}(s)-x_{i}\left(\gamma_{h}(s)\right)\right|\right) d s \\
& +\frac{L_{g}}{\Gamma \alpha} \int_{0}^{t}(t-s)^{\alpha-1} \sum_{1 \leq i, j \leq m}\left|c_{i j}\right|\left(\sum_{i=1}^{m}\left|x_{i}(s-\tau)-x_{i}\left(\gamma_{h}(s-\tau)\right)\right|\right) d s .
\end{aligned}
$$

Define $m(t)=\sup _{-\tau \leq s \leq t} \sum_{i=1}^{m}\left|\varepsilon_{i}^{h}(s)\right|$ and the module of continuity $w(x, h, T)=\max \left\{\sum_{i=1}^{m} \mid x_{i}(s)-\right.$ $\left.x_{i}\left(\gamma_{h}(s)\right) \mid:-\tau \leq s \leq T\right\}$. Therefore, we can write equation (4.22) for $t \geq 0$ as

$$
m(t) \leq u(t)+\frac{\beta}{\Gamma \alpha} \int_{0}^{t}(t-s)^{\alpha-1} m(s) d s,
$$

where

$$
\beta:=\sum_{i=1}^{m} a_{i}+\sum_{1 \leq i, j \leq m}\left(\left|b_{i j}\right| L_{f}+\left|c_{i j}\right| L_{g}\right)
$$

and

$$
u(t):=\frac{\beta t^{\alpha}}{\Gamma(\alpha+1)} w(x, h, T)
$$

Since $u(t)$ is non negative, non-decreasing and locally integrable function on $[0, T), \beta$ is a positive constant, then by Lemma $2.13,(2.17)$ holds, i.e.

$$
m(t) \leq \frac{\beta t^{\alpha}}{\Gamma(\alpha+1)} w(x, h, T) E_{\alpha, 1}\left(\beta t^{\alpha}\right), \quad t \in[0, T) .
$$

Using (2.7) we obtain

$$
m(t) \leq \frac{\beta t^{\alpha}}{\Gamma(\alpha+1)} w(x, h, T) e^{\beta t}, \quad t \in[0, T) .
$$

From the hypothesis of theorem, it follows that on $[0, T), \varepsilon_{i}^{h}(t) \rightarrow 0$ uniformly as $h \rightarrow 0$. We can summarize the above result as the following theorem. 
Theorem 4.3. Assume that the assumptions (A1) and (A2) holds. Then for every $h \in(0, r)$, the solution $x$ of the system (1.1) can be approximated using constant argument and the approximation satisfies:

$$
\left|x_{i}(t)-z_{i}^{h}(t)\right| \leq \frac{\beta t^{\alpha} e^{\beta t}}{\Gamma(\alpha+1)} w(x, h, T),
$$

where $\beta$ is given by (4.24) and $w(x, h, T)=\max \left\{\sum_{i=1}^{m}\left|x_{i}(s)-x_{i}\left(\gamma_{h}(s)\right)\right|:-\tau \leq s \leq T\right\}$. Furthermore, $z_{i}^{h}(t) \rightarrow x_{i}(t)$ uniformly as $h \rightarrow 0$, and

$$
\left|x_{i}(t)-z_{i}^{h}(t)\right| \leq \frac{\beta t^{\alpha} e^{\beta t} w(x, h, T)}{\Gamma(\alpha+1)}, \quad t \in[0, T) .
$$

On $[\tau, T)$ the functions $x_{i}$ and $z_{i}^{h}$ are Lipschitz functions, and hence

$$
w(x, h, T) \leq \operatorname{ch} \text { on }[\tau, T),
$$

with $c$ a positive constant. If the initial function $\varphi$ is Lipschitz on $[-\tau, 0]$, then $w(x, h, T) \leq c h$ on $[0, T)$.

Corollary 1. If the initial function $\varphi$ is Lipschitz on $[-\tau, 0]$, then the estimations in (4.25) and (4.26) become

$$
\begin{gathered}
\left|x_{i}(t)-z_{i}^{h}(t)\right| \leq \frac{\beta T^{\alpha} e^{\beta T}}{\Gamma(\alpha+1)} c h, \quad t \in[0, T), \\
\left|x_{i}(n h)-z_{i}^{h}(n h)\right| \leq \frac{\beta e^{[\beta+\ln (\alpha)] T}}{\Gamma(\alpha+1)} c h, \quad n \leq\left[\frac{T}{h}\right]
\end{gathered}
$$

and for $\alpha \rightarrow 1$ the result is also true.

The results are true for the systems purely ordinary and purely delayed.

Corollary 2. The assertion of Theorem 4.3 remains true if $f(\cdot) \equiv 0$, i.e. the $x$ solution of the system of differential equations

$$
\begin{aligned}
D_{t}^{\alpha} x_{i}(t) & =-a_{i} x_{i}(t)+\sum_{j=1}^{m} c_{i j} g\left(x_{j}(t-\tau)\right)+I_{i} \quad t \in[0, T) \\
x_{i}(t) & =\varphi_{i}(t), \quad t \in[-\tau, 0], \quad T<\infty, \quad i=1,2, \cdots, m .
\end{aligned}
$$

can be approximated using constant argument and the approximate satisfies:

$$
\begin{gathered}
\left|x_{i}(t)-z_{i}^{h}(t)\right| \leq \frac{\beta_{1} T^{\alpha} e^{\beta_{1} T}}{\Gamma(\alpha+1)} c h, \quad t \in[0, T), \\
\left|x_{i}(n h)-z_{i}^{h}(n h)\right| \leq \frac{\beta_{1} e^{\left[\beta_{1}+\ln (\alpha)\right] T}}{\Gamma(\alpha+1)} c h, \quad n \leq\left[\frac{T}{h}\right]
\end{gathered}
$$

and for $\alpha \rightarrow 1$ the result is also true, with $\beta_{1}=\sum_{i=1}^{m} a_{i}+\sum_{1 \leq i, j \leq m}\left|c_{i j}\right| L_{g}$. 
Corollary 3. The assertion of Theorem 4.3 remains true if $g(\cdot) \equiv 0$, i.e. the $x$ solution of the system of differential equations

$$
\begin{aligned}
D_{t}^{\alpha} x_{i}(t) & =-a_{i} x_{i}(t)+\sum_{j=1}^{m} b_{i j} f\left(x_{j}(t)\right)+I_{i} \quad t \in[0, T) \\
x_{i}(0) & =x_{i}^{0}, \quad i=1,2, \cdots, m .
\end{aligned}
$$

can be approximated using constant argument and the approximate satisfies:

$$
\begin{gathered}
\left|x_{i}(t)-z_{i}^{h}(t)\right| \leq \frac{\beta_{2} T^{\alpha} e^{\beta_{2} T}}{\Gamma(\alpha+1)} c h, \quad t \in[0, T), \\
\left|x_{i}(n h)-z_{i}^{h}(n h)\right| \leq \frac{\beta_{2} e^{\left[\beta_{2}+\ln (\alpha)\right] T}}{\Gamma(\alpha+1)} \text { ch, } n \leq\left[\frac{T}{h}\right]
\end{gathered}
$$

and for $\alpha \rightarrow 1$ the result is also true, with $\beta_{2}=\sum_{i=1}^{m} a_{i}+\sum_{1 \leq i, j \leq m}\left|b_{i j}\right| L_{f}$.

\section{$5 \quad$ Numerical Result}

In this section we present two numerical examples to validate the obtained theoretical results. However, before to present the details we note that the sequence $\mathfrak{a}_{i}^{h}$ in Theorem 4.1 can be rewritten. Indeed, we have that if $\mathfrak{a}_{i}^{h}(n)=z_{i}^{h}(n h)$, then the sequences satisfy the following difference equations

$$
\begin{aligned}
z_{i}^{h}(t)= & \mathfrak{a}_{i}^{h}(n)+\frac{1}{\Gamma \alpha} \int_{n h}^{t}(t-s)^{\alpha-1}\left(-a_{i} \mathfrak{a}_{i}^{h}(n)+\sum_{j=1}^{m} b_{i j} f\left(\mathfrak{a}_{j}^{h}(n)\right)\right. \\
& \left.+\sum_{j=1}^{m} c_{i j} g\left(\mathfrak{a}_{j}^{h}(n-\nu)\right)+I_{i}\right) d s .
\end{aligned}
$$

The above problem (5.1) is a discretization of the original system (1.1).

Example 5.1. Consider the following fractional-order bi-directional neural network without delay:

$$
\begin{aligned}
& D_{t}^{\alpha} x_{1}(t)=-x_{1}(t)+2 f\left(x_{2}(t)\right)+5 \\
& D_{t}^{\alpha} x_{2}(t)=-x_{2}(t)-1.5 f\left(x_{1}(t)\right)+10
\end{aligned}
$$

with $x_{1}(0)=0.5, x_{2}(0)=1$.

The activation function is $f(\cdot)=\tanh (\cdot)$.

In discrete form, we can write equation (5.2) as

$$
\begin{aligned}
\mathfrak{a}_{1}^{h}(n+1) & =\left(1-\frac{h^{\alpha}}{\Gamma(\alpha+1)}\right) \mathfrak{a}_{1}^{h}(n)+\frac{h^{\alpha}}{\Gamma(\alpha+1)}\left(2 f\left(\mathfrak{a}_{2}^{h}(n)+5\right)\right. \\
\mathfrak{a}_{2}^{h}(n+1) & =\left(1-\frac{h^{\alpha}}{\Gamma(\alpha+1)}\right) \mathfrak{a}_{2}^{h}(n)+\frac{h^{\alpha}}{\Gamma(\alpha+1)}\left(-1.5 f\left(\mathfrak{a}_{1}^{h}(n)+10\right)\right.
\end{aligned}
$$




\begin{tabular}{|c|c|c|c|c|}
\hline \multicolumn{5}{|c|}{ Actual versus Approximated solution } \\
\hline$t$ & $x_{1}(t)_{\text {actual }}$ & $x_{2}(t)_{\text {actual }}$ & $x_{1}(t)_{\text {approx }}$ & $x_{2}(t)_{\text {approx }}$ \\
\hline 0 & 0.5000 & 1.0000 & 0.5000 & 1.0000 \\
\hline 0.75 & 3.1427 & 4.8282 & 3.9266 & 4.9781 \\
\hline 1.50 & 5.0183 & 6.6172 & 4.7145 & 5.8738 \\
\hline 2.25 & 5.9837 & 7.5345 & 5.1331 & 6.3525 \\
\hline 3.00 & 5.9837 & 7.5345 & 5.4008 & 6.6591 \\
\hline 3.75 & 6.4788 & 8.0050 & 5.5892 & 6.8753 \\
\hline 5.25 & 6.8629 & 8.3698 & 5.8403 & 7.1638 \\
\hline 6.25 & 6.9297 & 8.4332 & 5.9550 & 7.2957 \\
\hline 8.50 & 6.9815 & 8.4824 & 6.1329 & 7.5004 \\
\hline 10.25 & 6.9951 & 8.4954 & 6.2271 & 7.6088 \\
\hline
\end{tabular}

For $\alpha=0.6, h=0.25$

\begin{tabular}{|c|c|c|c|c|}
\hline \multicolumn{5}{|c|}{ Actual versus Approximated solution } \\
\hline$t$ & $x_{1}(t)_{\text {actual }}$ & $x_{2}(t)_{\text {actual }}$ & $x_{1}(t)_{\text {approx }}$ & $x_{2}(t)_{\text {approx }}$ \\
\hline 0 & 0.5000 & 1.0000 & 0.5000 & 1.0000 \\
\hline 1.20 & 4.5899 & 6.1175 & 4.4832 & 5.6080 \\
\hline 2.40 & 5.6167 & 7.1327 & 5.2037 & 6.4324 \\
\hline 3.20 & 6.2061 & 7.7153 & 5.4630 & 6.7299 \\
\hline 4.40 & 6.5444 & 8.0496 & 5.7175 & 7.0223 \\
\hline 5.60 & 6.7385 & 8.2415 & 5.8869 & 7.2171 \\
\hline 7.20 & 6.9139 & 8.4149 & 6.0427 & 7.3964 \\
\hline 9.20 & 6.9716 & 8.4720 & 6.1754 & 7.5493 \\
\hline 10.60 & 6.9837 & 8.4839 & 6.2441 & 7.6284 \\
\hline 11.40 & 6.9906 & 8.4908 & 6.2772 & 7.6665 \\
\hline
\end{tabular}

For $\alpha=0.6, h=0.20$

\begin{tabular}{|c|c|c|c|c|}
\hline \multicolumn{5}{|c|}{ Actual versus Approximated solution } \\
\hline$t$ & $x_{1}(t)_{\text {actual }}$ & $x_{2}(t)_{\text {actual }}$ & $x_{1}(t)_{\text {approx }}$ & $x_{2}(t)_{\text {approx }}$ \\
\hline 0 & 0.5000 & 1.0000 & 0.5000 & 1.0000 \\
\hline 1.10 & 3.4040 & 4.6975 & 4.4079 & 5.5167 \\
\hline 1.50 & 3.4040 & 4.6975 & 4.7447 & 5.9030 \\
\hline 2.20 & 4.4136 & 5.7665 & 5.1324 & 6.3484 \\
\hline 3.10 & 5.1405 & 6.5349 & 5.4443 & 6.7071 \\
\hline 3.70 & 5.1405 & 6.5349 & 5.5904 & 6.8751 \\
\hline 5.20 & 6.0389 & 7.4844 & 5.8416 & 7.1643 \\
\hline 6.20 & 6.3091 & 7.7699 & 5.9562 & 7.2963 \\
\hline 8.50 & 6.6429 & 8.1226 & 6.1369 & 7.5046 \\
\hline 10.30 & 6.8154 & 8.3050 & 6.2324 & 7.6146 \\
\hline
\end{tabular}

For $\alpha=0.6, h=0.10$

TABLE 1. Actual versus Approximated solution for system (5.2) for $\alpha=0.6$

\begin{tabular}{|c|c|c|}
\hline$t$ & $\left|\varepsilon_{\left.x_{1}(t)\right)}\right|$ & $\left|\varepsilon_{x_{2}(t)}\right|$ \\
\hline 0 & 0 & 0 \\
\hline 0.75 & 0.7839 & 0.1499 \\
\hline 1.50 & 0.3038 & 0.7434 \\
\hline 2.25 & 0.8506 & 1.1820 \\
\hline 3.00 & 0.5829 & 0.8754 \\
\hline 3.75 & 0.8896 & 1.1297 \\
\hline 5.25 & 1.0226 & 1.206 \\
\hline 6.25 & 0.9747 & 1.1375 \\
\hline 8.50 & 0.8486 & 0.9820 \\
\hline 10.25 & 0.7680 & 0.8866 \\
\hline
\end{tabular}

For $\alpha=0.6, h=0.25$

\begin{tabular}{|c|c|c|}
\hline$t$ & $\left|\varepsilon_{x_{1}(t)}\right|$ & $\left|\varepsilon_{x_{2}(t)}\right|$ \\
\hline 0 & 0 & 0 \\
\hline 1.20 & 0.1067 & 0.5095 \\
\hline 2.40 & 0.4130 & 0.7003 \\
\hline 3.20 & 0.7431 & 0.9854 \\
\hline 4.40 & 0.8269 & 1.0273 \\
\hline 5.60 & 0.8516 & 1.0244 \\
\hline 7.20 & 0.8712 & 1.0185 \\
\hline 9.20 & 0.7962 & 0.9227 \\
\hline 10.60 & 0.7396 & 0.8555 \\
\hline 11.40 & 0.7134 & 0.8243 \\
\hline
\end{tabular}

For $\alpha=0.6, h=0.20$

\begin{tabular}{|c|c|c|}
\hline$t$ & $\left|\varepsilon_{\left.x_{1}(t)\right)}\right|$ & $\left|\varepsilon_{x_{2}(t)}\right|$ \\
\hline 0 & 0 & 0 \\
\hline 1.10 & 1.0039 & 0.8192 \\
\hline 1.50 & 1.3407 & 1.2055 \\
\hline 2.20 & 0.7188 & 0.5819 \\
\hline 3.10 & 0.3038 & 0.1722 \\
\hline 3.70 & 0.4499 & 0.3402 \\
\hline 5.20 & 0.1973 & 0.3201 \\
\hline 6.20 & 0.3529 & 0.4736 \\
\hline 8.50 & 0.5060 & 0.6180 \\
\hline 10.30 & 0.5830 & 0.6904 \\
\hline
\end{tabular}

For $\alpha=0.6, h=0.10$

TABLE 2. Error for different $h$ for system (5.2) 


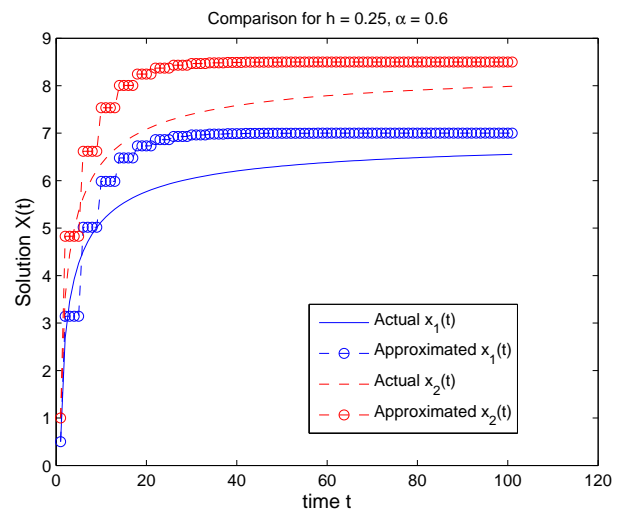

(a) For $\alpha=0.6, h=0.25$

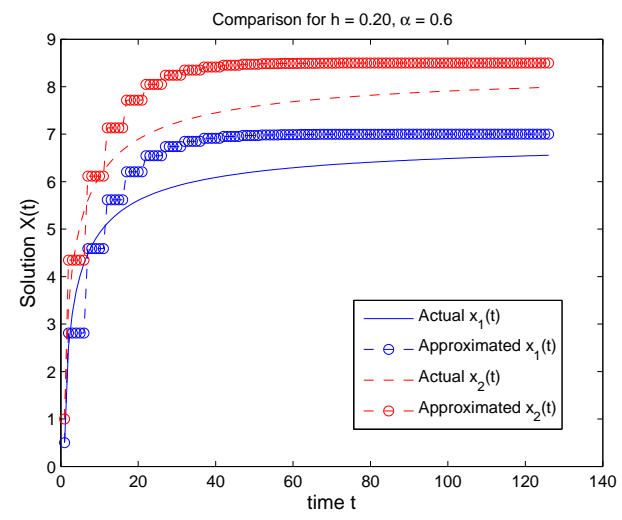

(b) For $\alpha=0.6, h=0.20$

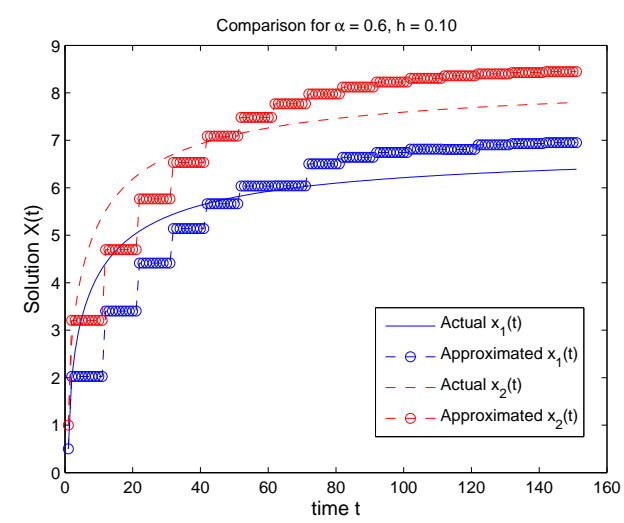

(c) For $\alpha=0.6, h=0.10$

FiguRE 1. Actual versus Approximated solution for system (5.2) for various $h$ with $\alpha=0.6$. 
The verification for results has been performed for different values of $h$ and $\alpha=0.6$. Table 1 and Figure 1(a)-1(c) containing solutions at different values of $h$ and $\alpha=0.6$ are given below. Table 2 illustrates the difference between the actual solution and approximated solution for different values of $h$, where absolute error $\varepsilon_{i}=\mid$ exact $_{i}-$ approximate $_{i} \mid, i=1,2$ for different values of $h$.

Comparing the above tables and figures, we can say that the solution of system (5.2) can be approximated through the discretized system. Since error is decreasing with increase in the value of $t$, thus the obtained numerical results are in support of theoretical analysis done in sub section 4 .

Now, for error estimation, we proceed as follows. In this case, $a_{1}=a_{2}=1, b_{11}=0, b_{12}=2, b_{21}=$ $-1.5, b_{22}=0, L_{f}=1$ and $c_{i j}=0$, for $1 \leq i, j \leq 2$. Thus for some $c>0$, using Corollary 3 , we have $\beta_{2}=\sum_{i=1}^{2} a_{i}+\sum_{1 \leq i, j \leq 2}\left|b_{i j}\right| L_{f}=5.5$. Then for $t \in[0, T), T<\infty$,

$$
\begin{aligned}
\left|x_{1}(t)-z_{1}^{h}(t)\right| & \leq \frac{5.5 T^{\alpha} e^{5.5 T}}{\Gamma(1+\alpha)} c h \\
\left|x_{2}(t)-z_{2}^{h}(t)\right| & \leq \frac{5.5 T^{\alpha} e^{5.5 T}}{\Gamma(1+\alpha)} c h
\end{aligned}
$$

and for $n \leq\left[\frac{T}{h}\right]$

$$
\begin{aligned}
&\left|x_{1}(n h)-z_{1}^{h}(n h)\right| \leq \frac{5.5 e^{[5.5+\ln (\alpha)] T}}{\Gamma(1+\alpha)} c h, \\
&\left|x_{2}(n h)-z_{2}^{h}(n h)\right| \leq \frac{5.5 e^{[5.5+\ln (\alpha)] T}}{\Gamma(1+\alpha)} c h .
\end{aligned}
$$

Varying $\alpha$ and $h$ for different values, we get different approximations for $x_{1}(t)$ and $x_{2}(t)$. Moreover, it is clear from (5.3) and (5.4) that $z_{i}^{h}(t) \rightarrow x_{i}(t)$ uniformly as $h \rightarrow 0$ supporting the theoretical analysis done in sub-section 4.2 .

Example 5.2. Consider the following fractional order delayed neural network model;

$$
\begin{aligned}
& D_{t}^{\alpha} x_{1}(t)=-x_{1}(t)+0.5 \tanh \left(x_{1}(t-\tau)\right)+10 \\
& D_{t}^{\alpha} x_{2}(t)=-x_{2}(t)+1.5 \tanh \left(x_{2}(t-\tau)\right)+20
\end{aligned}
$$

with initial conditions $(10,5)$ for $t \in[-\tau, 0]$. We discuss three different cases of $h$ for $\tau=0.05$ in this example.

Table 3 and Figures 2(a)-2(c) depict the comparison of solutions of system (5.5) for different values of $h$ for $\alpha=0.8$ and fixed delay $\tau=0.05$. Table 4 illustrates the difference between actual solution and approximated solution for different values of $h$, where absolute error is $\varepsilon_{i}=\mid$ exact $_{i}-$ approximate $_{i} \mid, i=1,2$ for different values of $h$. As $t$ is increasing, the difference between the actual solution and the approximated solution is decreasing. In other way, we can say that the approximated solution is converging to the actual solution.

For this example, error estimation proceeds as follows. In this case, $a_{1}=a_{2}=1, b_{i j}=0$, for $1 \leq i, j \leq 2$ and $c_{11}=0.5, c_{12}=0, c_{21}=0, c_{22}=1.5, L_{g}=1$. Thus for some $c>0$, using Corollary 


\begin{tabular}{|c|c|c|c|c|}
\hline \multicolumn{5}{|c|}{ Actual versus Approximated solution } \\
\hline$t$ & $x_{1}(t)_{\text {actual }}$ & $x_{2}(t)_{\text {actual }}$ & $x_{1}(t)_{\text {approx }}$ & $x_{2}(t)_{\text {approx }}$ \\
\hline 0 & 10.0000 & 5.0000 & 10.0000 & 5.0000 \\
\hline 1.20 & 10.4201 & 19.0764 & 10.0929 & 8.0666 \\
\hline 2.40 & 10.4313 & 19.3555 & 10.1327 & 9.3790 \\
\hline 3.20 & 10.4472 & 19.8532 & 10.1686 & 10.5632 \\
\hline 4.40 & 10.4590 & 20.2169 & 10.2010 & 11.6318 \\
\hline 5.60 & 10.4666 & 20.4524 & 10.2302 & 12.5959 \\
\hline 7.20 & 10.4730 & 20.6538 & 10.2803 & 14.2508 \\
\hline 9.20 & 10.4781 & 20.8141 & 10.3211 & 15.5981 \\
\hline 10.60 & 10.4806 & 20.8926 & 10.3386 & 16.1747 \\
\hline 11.40 & 10.4818 & 20.9293 & 10.3544 & 16.6950 \\
\hline
\end{tabular}

For $\alpha=0.8, h=0.05$

\begin{tabular}{|c|c|c|c|c|}
\hline \multicolumn{5}{|c|}{ Actual versus Approximated solution } \\
\hline$t$ & $x_{1}(t)_{\text {actual }}$ & $x_{2}(t)_{\text {actual }}$ & $x_{1}(t)_{\text {approx }}$ & $x_{2}(t)_{\text {approx }}$ \\
\hline 0 & 10.0000 & 5.0000 & 10.0000 & 5.0000 \\
\hline 1.20 & 10.0851 & 7.8083 & 10.0378 & 7.6659 \\
\hline 2.40 & 10.2143 & 12.0720 & 10.1009 & 9.7467 \\
\hline 3.20 & 10.2629 & 13.6769 & 10.1576 & 11.6198 \\
\hline 4.40 & 10.3033 & 15.0084 & 10.2106 & 13.3696 \\
\hline 5.60 & 10.3368 & 16.1132 & 10.2611 & 15.0331 \\
\hline 7.20 & 10.3876 & 17.7909 & 10.3415 & 17.3537 \\
\hline 9.20 & 10.4226 & 18.9460 & 10.3880 & 18.5654 \\
\hline 10.60 & 10.4358 & 19.3807 & 10.4016 & 18.8685 \\
\hline 11.40 & 10.4467 & 19.7414 & 10.4101 & 19.0200 \\
\hline
\end{tabular}

For $\alpha=0.8, h=0.10$

\begin{tabular}{|c|c|c|c|c|}
\hline \multicolumn{5}{|c|}{ Actual versus Approximated solution } \\
\hline$t$ & $x_{1}(t)_{\text {actual }}$ & $x_{2}(t)_{\text {actual }}$ & $x_{1}(t)_{\text {approx }}$ & $x_{2}(t)_{\text {approx }}$ \\
\hline 0 & 10.0000 & 5.0000 & 10.0000 & 5.0000 \\
\hline 1.20 & 10.5280 & 24.4866 & 10.2524 & 13.3293 \\
\hline 2.40 & 10.5209 & 21.6558 & 10.3258 & 15.7503 \\
\hline 3.20 & 10.3976 & 17.8010 & 10.3744 & 17.4539 \\
\hline 4.40 & 10.4403 & 20.0968 & 10.4137 & 18.6528 \\
\hline 5.60 & 10.4983 & 21.5996 & 10.4393 & 19.4964 \\
\hline 7.20 & 10.4604 & 20.2626 & 10.4699 & 20.5078 \\
\hline 9.20 & 10.4838 & 21.0791 & 10.4851 & 21.0087 \\
\hline 10.60 & 10.4787 & 20.8650 & 10.4895 & 21.1543 \\
\hline 11.40 & 10.4792 & 20.9018 & 10.4926 & 21.2567 \\
\hline
\end{tabular}

For $\alpha=0.8, h=0.20$

TABLE 3. Actual versus Approximated solution for $\tau=0.05$ for system (5.5)

\begin{tabular}{|c|c|c|}
\hline$t$ & $\left|\varepsilon_{x_{1}(t)}\right|$ & $\left|\varepsilon_{x_{2}(t)}\right|$ \\
\hline 0 & 0 & 0 \\
\hline 1.20 & 0.3272 & 11.0098 \\
\hline 2.40 & 0.2986 & 9.9765 \\
\hline 3.20 & 0.2786 & 9.2900 \\
\hline 4.40 & 0.2580 & 8.5851 \\
\hline 5.60 & 0.2364 & 7.8565 \\
\hline 7.20 & 0.1927 & 6.4030 \\
\hline 9.20 & 0.1570 & 5.2160 \\
\hline 10.60 & 0.1420 & 4.7179 \\
\hline 11.40 & 0.1274 & 4.2343 \\
\hline
\end{tabular}

For $\alpha=0.8, h=0.05, \tau=0.05$

\begin{tabular}{|c|c|c|}
\hline$t$ & $\left|\varepsilon_{x_{1}(t)}\right|$ & $\left|\varepsilon_{x_{2}(t)}\right|$ \\
\hline 0 & 0 & 0 \\
\hline 1.20 & 0.0473 & 0.1424 \\
\hline 2.40 & 0.1134 & 2.3253 \\
\hline 3.20 & 0.1053 & 2.0571 \\
\hline 4.40 & 0.0927 & 1.6388 \\
\hline 5.60 & 0.0757 & 1.0801 \\
\hline 7.20 & 0.0461 & 0.4372 \\
\hline 9.20 & 0.0346 & 0.3806 \\
\hline 10.60 & 0.0342 & 0.5122 \\
\hline 11.40 & 0.0366 & 0.7214 \\
\hline
\end{tabular}

For $\alpha=0.8, h=0.10, \tau=0.05$

\begin{tabular}{|c|c|c|}
\hline$t$ & $\left|\varepsilon_{\left.x_{1}(t)\right)}\right|$ & $\left|\varepsilon_{x_{2}(t)}\right|$ \\
\hline 0 & 0 & 0 \\
\hline 1.20 & 0.2756 & 11.1573 \\
\hline 2.40 & 0.1951 & 5.9055 \\
\hline 3.20 & 0.0232 & 0.3471 \\
\hline 4.40 & 0.0266 & 1.4440 \\
\hline 5.60 & 0.0590 & 2.1032 \\
\hline 7.20 & 0.0095 & 0.2452 \\
\hline 9.20 & 0.0013 & 0.0704 \\
\hline 10.60 & 0.0108 & 0.2893 \\
\hline 11.40 & 0.0134 & 0.3549 \\
\hline
\end{tabular}

For $\alpha=0.8, h=0.20, \tau=0.05$

TABLE 4. Error for $\alpha=0.8, \tau=0.05$ for system (5.5) 


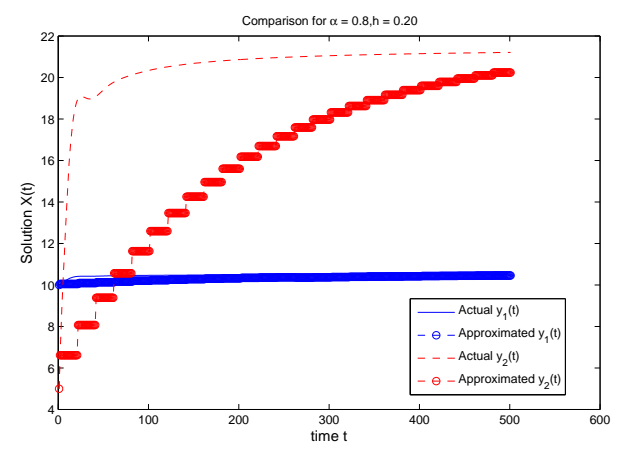

(a) For $\alpha=0.8, h=0.05$

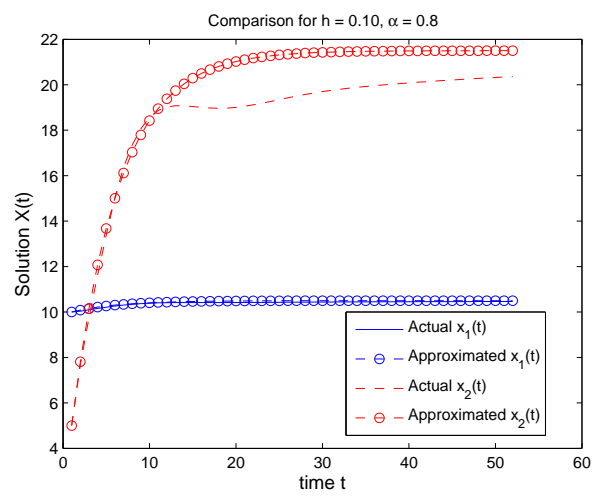

(b) For $\alpha=0.8, h=0.10$

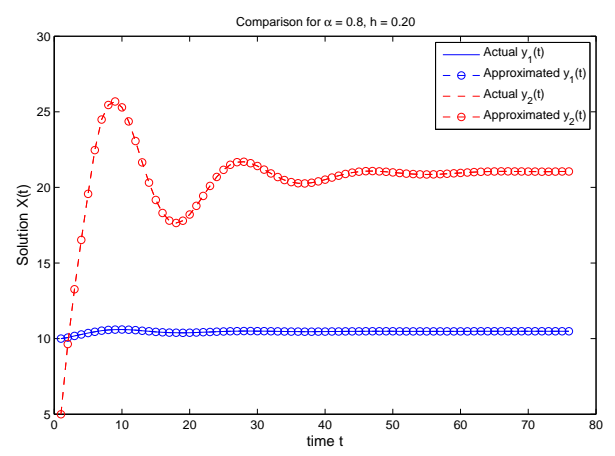

(c) For $\alpha=0.8, h=0.20$

FiguRE 2. Actual versus Approximated solution for system (5.5) with $\alpha=0.8, \tau=0.05$ 
2 , we have $\beta_{1}=\sum_{i=1}^{2} a_{i}+\sum_{1 \leq i, j \leq 2}\left|c_{i j}\right| L_{g}=4$. Then for $t \in[0, T), T<\infty$, we can give error estimate as

$$
\begin{aligned}
\left|x_{1}(t)-z_{1}^{h}(t)\right| \leq c h \frac{4}{\Gamma(1+\alpha)} T^{\alpha} e^{4 t} \\
\left|x_{2}(t)-z_{2}^{h}(t)\right| \leq \operatorname{ch} \frac{4}{\Gamma(1+\alpha)} T^{\alpha} e^{4 t} .
\end{aligned}
$$

and for $n \leq\left[\frac{T}{h}\right]$

$$
\begin{aligned}
& \left|x_{1}(n h)-z_{1}^{h}(n h)\right| \leq \operatorname{ch} \frac{4}{\Gamma(1+\alpha)} e^{(4+\ln (0.8) T}, \\
& \left|x_{2}(n h)-z_{2}^{h}(n h)\right| \leq \operatorname{ch} \frac{4}{\Gamma(1+\alpha)} e^{(4+\ln (0.8) T} .
\end{aligned}
$$

In this case also, by varying $h$ for different values, we can obtain various estimates for $x_{1}(t)$ and $x_{2}(t)$ and clearly from (5.6) and (5.7), $z_{i}^{h}(t) \rightarrow x_{i}(t)$ uniformly as $h \rightarrow 0$ supporting the theoretical analysis done in sub-section 4.2 .

\section{Conclusion}

This work is devoted to study Mittag-Leffler stability for a delayed fractional-order cellular neural network containing along with the approximation of solution of model system (1.1) using discretization scheme. Later, it is proved that the solution of the original problem can be approximated for large $t$ in a bounded interval $[0, T)$ using discretization with piecewise constant arguments of generalized type. Furthermore, the error function is estimated which establishes the uniform convergence of the fractional polygonal lines of order $\alpha$ as $h$ tends to zero. Under the obtained sufficient conditions, the discrete-time analogue preserves the unique equilibrium of the continuous-time network. Moreover, the global Mittag-Leffler stability and hence global asymptotic stability indicates an improvement over the uniform stability of the continuous-time network established in [20,21]. Hence the proposed study can be used to describe some essential aspects of fractional calculus in delayed and non-delayed neural network model system for approximating the solutions.

\section{Acknowledgement}

We are thankful to the reviewers for their valuable comments and suggestions.

S. Abbas thanks for the support of DST-SERB, IITM-FDE/SYA/14 and M. Pinto thanks for the support of FONDECYT 1120709. D. Sepúlveda thanks for the support of research project CIR 1418 at Universidad Central de Chile.

\section{References}

[1] Sabatier, J.; Poullain, S.; Latteux, P.; Oustaloup, A. Robust speed control of a low damped electromechanical system based on CRONE control: Application to a four mass experimental 
test bench. Nonlinear Dynamics 38.1-4 (2004): 383-400.

[2] Caputo, M.; Mainardi, F. A new dissipation model based on memory mechanism. Pure and Applied Geophysics 91.1 (1971): 134-147.

[3] Anastasio, T.J. The fractional-order dynamics of brainstem vestibulo-oculomotor neurons. Biological Cybernetics 72.1 (1994): 69-79.

[4] Mainardi, F.; Raberto, M.; Gorenflo, R.; Scalas, E. Fractional calculus and continuous-time finance II: the waiting-time distribution. Physica A: Statistical Mechanics and its Applications 287.3 (2000): 468-481.

[5] Celik, V.; Yakup, D. Chaotic fractional order delayed cellular neural network. New Trends in Nanotechnology and Fractional Calculus Applications. Springer Netherlands (2010): 313-320.

[6] Chen, L.; Chai, Y.; Wu, R.; Ma, T.; Zhai, H. Dynamic analysis of a class of fractional-order neural networks with delay. Neurocomputing 111 (2013): 190-194.

[7] Wang, H.; Yongguang, Y.; Guoguang, W. Stability analysis of fractional-order Hopfield neural networks with time delays. Neural Networks 55 (2014): 98-109.

[8] Stamova, I. Global Mittag-Leffler stability and synchronization of impulsive fractional-order neural networks with time-varying delays. Nonlinear Dynamics 77.4 (2014): 1251-1260.

[9] Deng, W.; Changpin, L.; Jinhu, L. Stability analysis of linear fractional differential system with multiple time delays. Nonlinear Dynamics 48.4 (2007): 409-416.

[10] Sierociuk, D.; Grzegorz, S.; Andrzej, D. Discrete fractional order artificial neural network. Acta Mechanica et Automatica 5 (2011): 128-132.

[11] Hirsch, M.W. Convergent activation dynamics in continuous time networks. Neural Networks 2.5 (1989): 331-349.

[12] Delavari, H.; Dumitru, B.; Jalil, S. Stability analysis of Caputo fractional-order nonlinear systems revisited. Nonlinear Dynamics 67.4 (2012): 2433-2439.

[13] Guo, S.; Lihong, H. Stability of nonlinear waves in a ring of neurons with delays. Journal of Differential Equations 236.2 (2007): 343-374.

[14] Zhang, S.; Yu, Y.; Wang, H. Mittag-Leffler stability of fractional-order Hopfield neural networks. Nonlinear Analysis: Hybrid Systems 16 (2015): 104-121.

[15] Kilbas, A.A.; Srivastava, H.M.; Trujillo, J.J. Theory and applications of fractional differential equations. Elsevier Science Limited 204 (2006).

[16] Miller, K.S.; Ross, B. An introduction to the fractional calculus and fractional differential equations. New York: Wiley (1993).

[17] Podlubny, I. Fractional differential equations: an introduction to fractional derivatives, fractional differential equations, to methods of their solution and some of their applications 198. Academic Press (1998). 
[18] Henry, D. Geometric theory of Semilinear Parabolic Partial Differential Equations. SpringerVerlag, Berlin-New York (1989).

[19] Wu, R.C.; Hei, X.D.; Chen, L.P. Finite-time stability of fractional-order neural networks with delay. Communications in Theoretical Physics 60 (2013): 189-193.

[20] Yang, X.; Song, Q.; Liu, Y.; Zhao, Z. Uniform stability analysis of fractional-order BAM neural networks with delays in the leakage terms. Abstract and Applied Analysis (2014). Hindawi Publishing Corporation.

[21] Chen, L.; Chai, Y.; Wu, R.; Ma, T.; Zhai, H. Dynamic analysis of a class of fractional-order neural networks with delay. Neurocomputing 111 (2013): 190-194.

[22] Ke, Y.; Miao, C. Stability analysis of fractional-order CohenGrossberg neural networks with time delay. International Journal of Computer Mathematics 92.6 (2015): 1102-1113.

[23] Abbas, S. Existence and attractivity of k-pseudo almost automorphic sequence solution of a model of bidirectional neural networks. Acta Applicandae Mathematicae 119.1 (2012): 57-74.

[24] Abbas, S.; Xia, Y. Existence and attractivity of k-almost automorphic sequence solution of a model of cellular neural networks with delay. Acta Mathematica Scientia 33.1 (2013): 290-302.

[25] Abbas, S. Pseudo almost periodic sequence solutions of discrete time cellular neural networks. Nonlinear Analysis, Modeling and Control 14.3 (2009): 283-301.

[26] Chiu K.S.; Pinto, M.; Jeng J.C. Existence and global convergence of periodic solutions in recurrent neural network models with a general piecewise alternately advanced and retarded argument. Acta Applicandae Mathematicae 136.1 (2015): 193-216.

[27] Pinto, M. Asymptotic equivalence of nonlinear and quasi linear differential equations with piecewise constant arguments. Mathematical and Computer Modelling 49 (2009): 451-458.

[28] Pinto, M.; Robledo G. Controllability and observability for a linear time varying system with piecewise constant delay. Acta Applicandae Mathematicae 136.1 (2015): 193-216.

[29] Huang, Z.; Wang, X.; Xia, Y. Exponential attractor of -almost periodic sequence solution of discrete-time bidirectional neural networks. Simulation Modelling Practice and Theory 18.3 (2010): 317-337.

[30] El-Sayed, A.M.A.; Salman, S.M. On a discretization process of fractional-order Riccati differential equation. Journal of Fractional Calculus and Applications 4.2 (2013): 251-259

[31] Raheem, ZF. El.; Salman, S.M. On a discretization process of fractional-order Logistic differential equation. Journal of the Egyptian Mathematical Society 22.3 (2014): 407-412.

[32] Agarwal, R.P.; El-Sayed, A.M.A.; Salman, S.M. Fractional-order Chuas system: discretization, bifurcation and chaos. Advances in Difference Equations (2013): 320.

[33] Ye, H.; Gao, J.; Ding, Y. A generalized Gronwall inequality and its application to a fractional differential equation. Journal of Mathematical Analysis and Applications 328.2 (2007): 10751081. 